\title{
A virtual care innovation for home mechanical ventilation
}

\author{
Reshma Amin MD, Regina Pizzuti RRT BA, Francine Buchanan MLIS, Louise Rose PhD MN
}

Cite as: CMAJ 2021 April 26;193:E607-11. doi: 10.1503/cmaj.202584

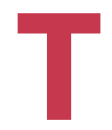
he Long-Term In-Home Ventilator Engagement (LIVE) program is a new virtual care program, rolled out during the coronavirus disease 2019 (COVID-19) pandemic to enable specialized centres to provide virtual support to people using home mechanical ventilation (HMV). At present, 251 patients from 8 hospital HMV programs (pediatric and adult) in Ontario are participating in the LIVE program.

Home mechanical ventilation is one of the most advanced, highly specialized and complex long-term therapies offered to patients outside of a hospital setting. In previous work using health administrative databases, our group estimated the prevalence of HMV in Canada to be $12.9 / 100000$ people. ${ }^{1}$ Patients are frequent users of the health care system, with high associated costs. Within 12 months of HMV initiation, $49.9 \%$ of patients have an emergency department visit, $29.9 \%$ are admitted to hospital, $43.9 \%$ use home care services and only $75.3 \%$ survive 1 year. ${ }^{2}$ The estimated median monthly public and private health care costs are $\$ 8733$ (interquartile range [IQR] \$5868-\$15 274) for patients who are invasively ventilated and \$3925 (IQR \$1212-\$7390) for patients living at home on noninvasive ventilation. ${ }^{3}$

The COVID-19 pandemic has been an unparalleled threat to the Canadian health care system. ${ }^{4}$ Users of home mechanical ventilation are at an increased risk of worsening respiratory failure and death secondary to severe acute respiratory syndrome coronavirus 2 (SARS-CoV-2), given their ongoing chronic respiratory insufficiency. ${ }^{5,6}$ Admitting HMV users to hospital uses scarce hospital ventilators needed for others - and possibly very difficult decisions about who should receive ventilation. ${ }^{7}$ Patients on HMV are particularly fearful of exposure to SARS-CoV-2 and avoid travel within the community, including for follow-up visits at the hospital. Virtual care offers a solution that enables patient- and family-centred care, facilitates knowledge sharing across health care sectors, ensures timely access to clinicians with the needed expertise and overcomes distance, cost and time constraints. ${ }^{8}$ The COVID-19 pandemic presented a pressing need for an innovative, virtual telehealth program for HMV users to keep these patients well and in their homes.

\section{KEY POINTS}

- The Long-Term In-Home Ventilator Engagement (LIVE) program in Ontario offers a multicomponent e-health intervention that enables secure, virtual home visits, customizable care plans and clinical workflows for respiratory symptoms and signs, as well as ventilator issues, telemonitoring of oximeters and ventilators and an integrated resource library.

- To date, the LIVE program has supported 251 children and adults using home mechanical ventilation from 8 programs in Ontario.

- Patients and families report feeling "connected, empowered and safe," and health care providers remark about the "ease of connection with families, benefits of the program for troubleshooting ventilator issues and improved clinical care."

\section{What is the Long-Term In-Home Ventilator Engagement (LIVE) program?}

The LIVE program is an intensive clinical management program delivered via an e-health platform (aTouchAway, Aetonix), creating a circle of care that includes HMV clinical teams, ventilator technology support specialists (the Ontario Ventilator Equipment Pool [ON-VEP]), the patient and their family caregivers. The LIVE program was codesigned by patients, families, health care professionals and clinical academics, in collaboration with our e-health partner. By bringing the expertise of HMV specialists virtually into the home, LIVE overcomes barriers related to distance and travel for patients who are often fragile and dependent on complex equipment that is hard to transport, as well as the risk of exposure to SARS-CoV-2. The standard of care before COVID-19 included in-person clinic visits with the specialized HMV team every 3-6 months for clinical assessment and ventilator data downloads. In addition, a respiratory therapist from the ON-VEP is available at all hours for equipment-related issues. The LIVE program comprises a multicomponent intervention that includes virtual home visits, customizable care plans and clinical workflows for respiratory symptoms and signs. It also addresses ventilator issues. The platform enables telemonitoring of oximeters and ventilators, 
as well as secure communication via messaging, audio and video calls among patients, families and health care providers (Table 1, Figure 1, Figure 2). Although other telemonitoring programs have been established and evaluated, ${ }^{9}$ at present, we are not aware of similar e-health interventions for HMV users in Canada or other countries.
When the incidence of SARS-CoV-2 infections started increasing in Canada, we were developing the LIVE virtual telehealth intervention for a randomized controlled trial funded by the Canadian Institutes of Health Research. The pandemic necessitated a rapid pivot to an early clinical rollout by 8 HMV programs.

\section{Table 1: The telehealth components of the LIVE program ${ }^{9}$}

\section{Component}

Telemedicine

Telecommunications

Teleconsultation

Telemonitoring

Teletherapy

Teleconference

Telecoaching

\section{Description}

HMV and ON-VEP team members use information from secure messaging, workflows and ventilator data downloads to provide clinical management

Two-way secure messaging via text, voice or video between patients, families and the health care team

Ad hoc consultation initiated by patients and families and provided by HMV and ON-VEP team members for management of clinical symptoms or ventilator troubleshooting

\section{Cellular connectivity and Bluetooth transmission of ventilator data downloads}

Ad hoc consultations and interpretation of ventilator data downloads can lead to changes in prescribed ventilator settings Three-way audio and video calls that can be initiated by patients, families or the health care team

Counselling and troubleshooting that is provided by a member of the HMV team if the ventilator data downloads show poor adherence to therapy

Note: $\mathrm{HMV}$ = home mechanical ventilation, LIVE = Long-term In Home Ventilator Engagement, ON-VEP = Ontario Ventilator Equipment Pool.

Two-way communication system allows patients, families or ventilator team members to initiate a conversation over text, audio or video chat.
Routine virtual clinic visits mirror in-person appointments. During these visits, a patient's physician reviews their respiratory technology use and ventilator settings, and answers any questions or concerns they may have about their ventilator.
Agile surveillance questionnaires are completed bimonthly and monthly. Questionnaires are used to monitor a patient's symptoms and ventilator issues.

Patients are coded as status green, yellow, or red based on their responses, which are then reviewed by the health care team and addressed with the family.
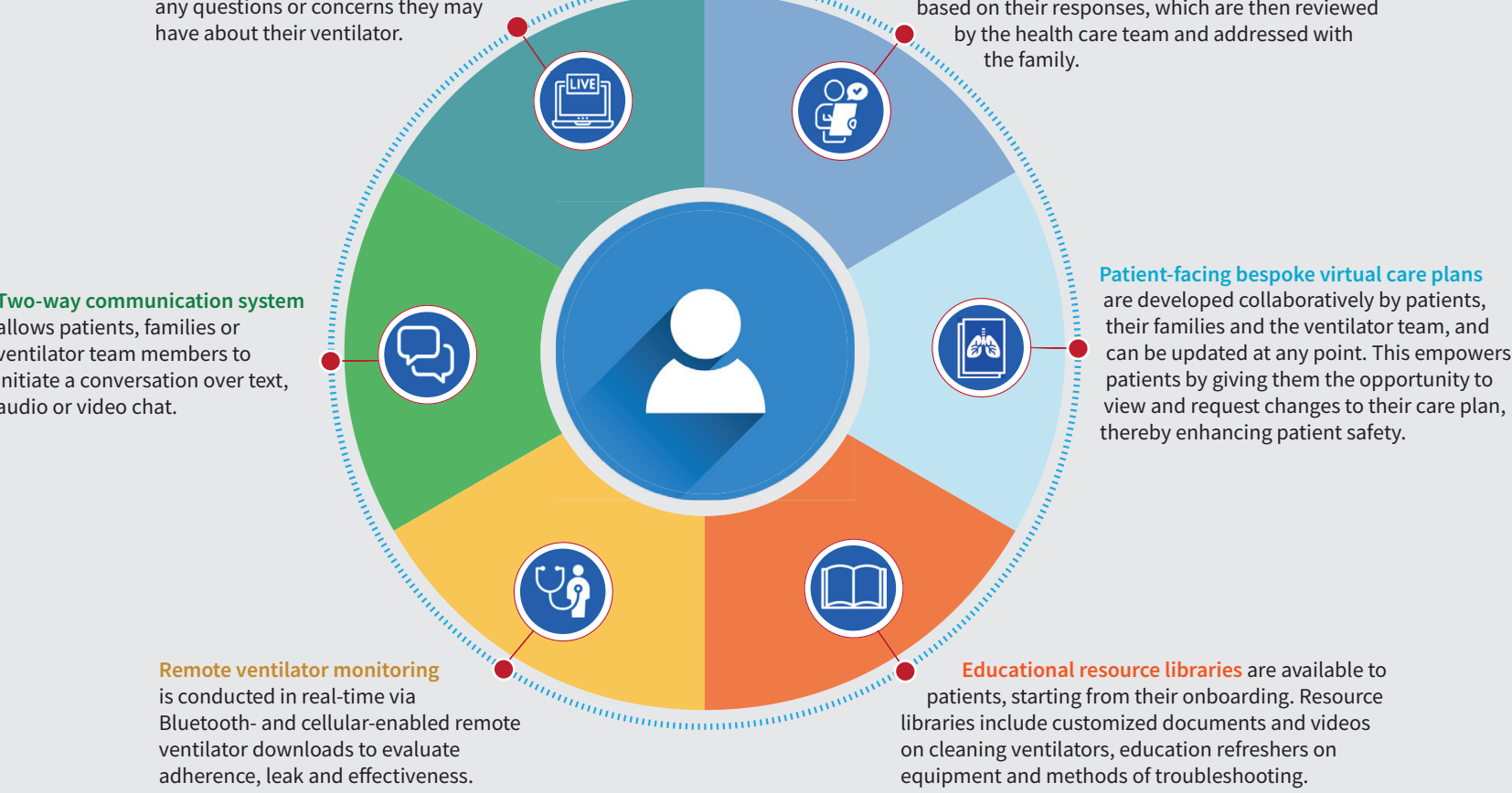

Figure 1: The 6 pillars of the Long-Term In-Home Ventilator Engagement (LIVE) program. 


\section{How is the LIVE program delivered and who is eligible?}

Specialist HMV teams identify patients who are at high risk of respiratory exacerbation (i.e., patients who are invasively ventilated and have a rapidly progressive underlying condition and/ or are medically fragile). With the support of the ON-VEP, the program app is downloaded onto the patient's personal device (or that of a parent or caregiver). If patients do not have a personal device compatible with the app or have inadequate Internet access, we loan patients a tablet with a subscriber identification module (SIM) card. The patient is then onboarded and oriented to the LIVE program. A member of the LIVE team connects with the patient via the app to review its functionality and the components of the LIVE program, and to guide the patient and their caregivers through completion of the virtual care plan. Once this process is complete, the patient appears on the HMV team's patient dashboard and two-way clinical engagement can begin. At present, there are 251 patients from
8 hospital HMV programs (pediatric and adult) in Ontario participating in the LIVE program (Figure 3).

\section{What are the unintended consequences of the LIVE program's rapid rollout?}

Four main issues arose with the rapid clinical rollout of the LIVE program. First, HMV patients in Ontario did not have equal access to LIVE. Only the $8 \mathrm{HMV}$ programs recruited for the randomized controlled trial that were already familiar with the app could be included. Second, privacy, security and legal requirements for virtual telehealth programs were not standardized across institutions, leading to contract delays and limiting the number of patients enrolled at some centres. Third, the rapid rollout meant that electronic health record integration was not feasible, resulting in some duplicate documentation. Fourth, although we made efforts to overcome digital inequality by providing tablets and SIM cards, we could only include patients able to speak English or French, given limited access to interpreters for virtual encounters.
A) Seamless 2-way communication between patients, families and health care providers.

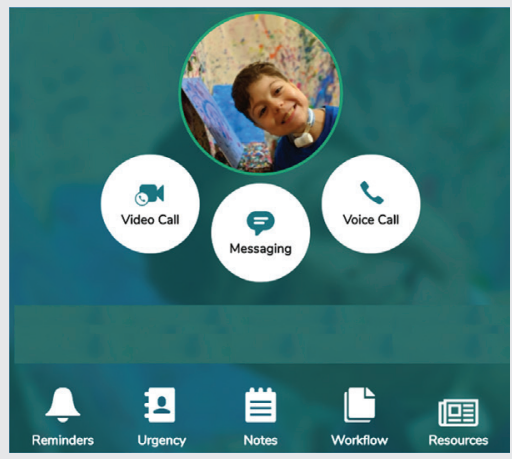

C) Intensive patient monitoring via questionnaires and ventilator downloads.
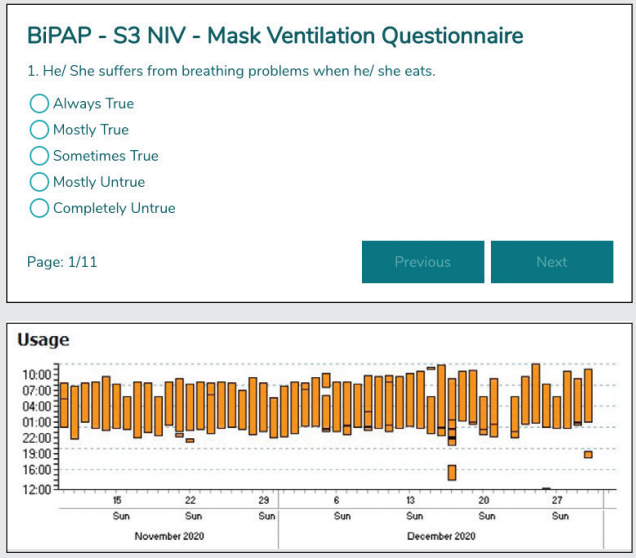

B) Virtual care plan that is collaboratively created and updated by patients, families and health care providers.

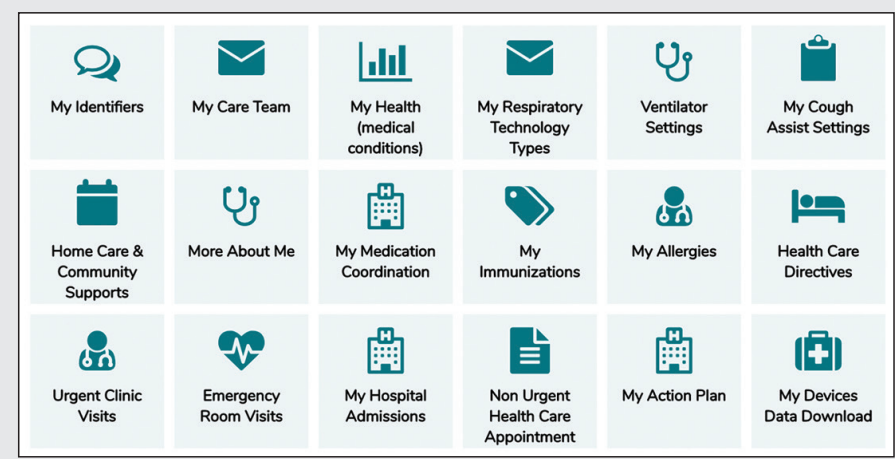

D) Education resource library, including documents and videos providing step-by-step instructions for equipment usage, cleaning and troubleshooting.

Oxygen saturation monitoring: Frequently Asked Questions

How does an oximeter work?

During use, the oximeter continuously calculates and gives a reading of the oxygen saturation and the heart rate. The beep's pitch drops as oxygen saturation falls and rises as it recovers. This is an important safety feature, as it allows you to hear changes in your child's oxygen saturation immediately without needing to look at the monitor all the time.

The probe you place on your child has a small red light on one side and a detector on the other side. The red light shines through your child's finger or toe and is seen by the detector on the other side. The detector measures the amount of oxygen in the blood.

For the oximeter to function, the sensor must be placed where a pulse can be detected, such as on the finger, foot, toe or earlobe.

Figure 2: Screen captures showing the features of the Long-Term In-Home Ventilator Engagement (LIVE) program. Reproduced with permission of Aetonix. 


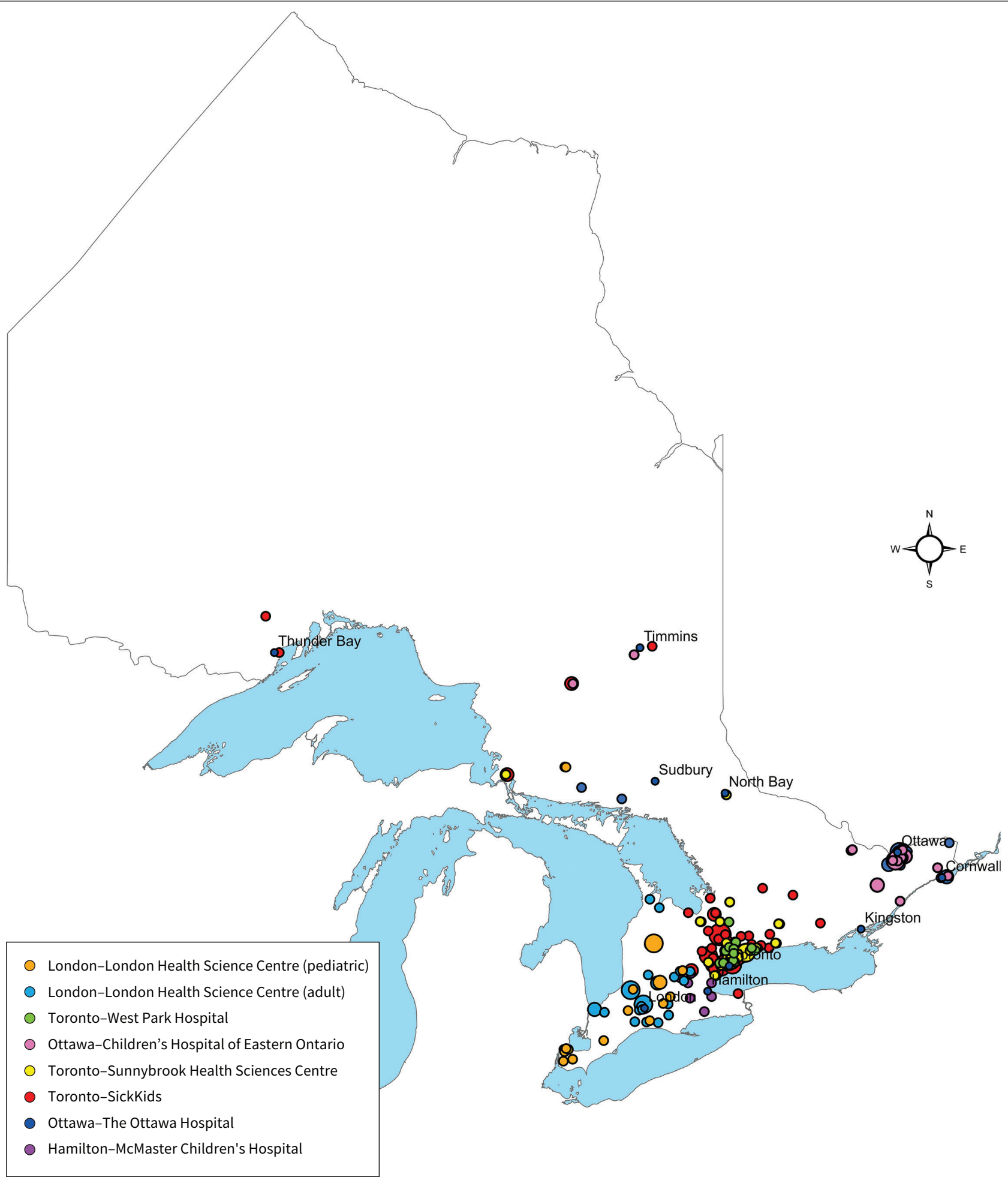

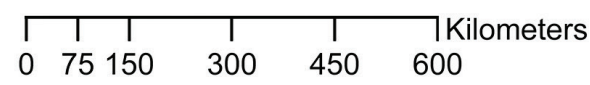

Figure 3: Geographic distribution of 251 partipants of LIVE program by forward sortation area (FSA) centroid. Map prepared by and reproduced with permission of Tom Fisher RRT, Feb. 16, 2021. 


\section{What is the evidence so far for the program?}

In other settings, virtual care for HMV users has been found to promote the efficiency of scarce health care resources by reducing emergent health care use and the cost of care to patients, family members, and the health system. ${ }^{10-13}$ To date, patients and family caregivers enrolled in LIVE report that the program engenders feelings of being "connected, empowered and safe." Qualitative feedback from HMV teams show the ease of connection with families, as well as the time-saving and clinical benefits of ventilator telemonitoring. The program has also helped prevent acute care visits, as illustrated in the case of an 11-year old girl who had been prescribed noninvasive ventilation. She completed our weekly questionnaire, reporting intermittent oxygen desaturations overnight, despite feeling well. The HMV team was able to obtain a remote ventilator data download showing the need for an increased target alveolar ventilation setting. These changes were made over the phone and, once implemented, resolved the issue. This patient would have otherwise been managed with a visit to an urgent clinic or the emergency department. Our group will generate formal evidence of the LIVE program using a mixed methods evaluation, including feasibility and acceptability.

\section{What can be expected in the future?}

The COVID-19 pandemic has changed the landscape of clinical medicine and health service delivery. As such, we anticipate that virtual care will remain an integral component of the clinical toolkit for the HMV population. Our lessons learned from the LIVE program clinical rollout and our randomized controlled trial (commencing recruitment in early 2021) can be leveraged to support a national scale-up and evaluation of virtual care for patients using HMV. In addition, opportunities exist to support integration with the electronic health record and to translate the provincial experience of the LIVE program to other complex and vulnerable patient populations living in the community, as well as in long-term care.

\section{References}

1. Rose L, McKim DA, Katz SL, et al. Home mechanical ventilation in Canada: a national survey. Respir Care 2015;60:695-704.

2. Povitz M, Rose L, Shariff SZ, et al. Home mechanical ventilation: a 12-year population-based retrospective cohort study. Respir Care 2018;63:380-7.

3. Nonoyama ML, McKim DA, Road J, et al. Healthcare utilisation and costs of home mechanical ventilation. Thorax 2018 Jan. 26 [Epub ahead of print]. doi: 10.1136/thoraxjnl-2017-211138.

4. Shoukat A, Wells CR, Langley JM, et al. Projecting demand for critical care beds during COVID-19 outbreaks in Canada. CMAJ 2020;192:E489-96.
5. Sanchez-Ramirez DC, Mackey D. Underlying respiratory diseases, specifically COPD, and smoking are associated with severe COVID-19 outcomes: a systematic review and meta-analysis. Respir Med 2020;171:106096.

6. Sanyaolu A, Okorie C, Marinkovic A, et al. Comorbidity and its impact on patients with COVID-19. SN Compr Clin Med 2020 June 25 [Epub ahead of print]. 1-8. doi: 10.1007/s42399-020-00363-4.

7. Barrett K, Khan YA, Mac S, et al. Estimation of COVID-19-induced depletion of hospital resources in Ontario, Canada. CMAJ 2020;192:E640-6.

8. Jamieson $\mathrm{T}$, Wallace R, Armstrong $\mathrm{K}$, et al. Virtual care: a framework for a patient-centric system. Toronto: Women's College Hospital; 2015. Available: www.womenscollegehospital.ca/assets/pdf/wihv/WIHV_VirtualHealthSymposium .pdf (accessed 2021 Feb. 15).

9. Ambrosino N, Vitacca M, Dreher M, et al.; ERS Tele-Monitoring of VentilatorDependent Patients Task Force. Tele-monitoring of ventilator-dependent patients: a European Respiratory Society Statement. Eur Respir J 2016;48: 648-63.

10. Muñoz-Bonet JI, López-Prats JL, Flor-Macián EM, et al. Usefulness of telemedicine for home ventilator-dependent children. J Telemed Telecare 2020;26:207-15.

11. Lopes de Almeida JP, Pinto AC, Pereira J, et al. Implementation of a wireless device for real-time telemedical assistance of home-ventilated amyotrophic lateral sclerosis patients: a feasibility study. Telemed J E Health 2010;16:883-8.

12. Bertini S, Picariello M, Gorini M, et al. Telemonitoring in chronic ventilatory failure: a new model of survellaince, a pilot study. Monaldi Arch Chest Dis 2012; 77:57-66.

13. Vitacca M, Bianchi L, Guerra A, et al. Tele-assistance in chronic respiratory failure patients: a randomised clinical trial. Eur Respir J 2009;33:411-8.

\section{Competing interests: None declared.}

This article has been peer reviewed.

The authors have obtained patient consent.

Affiliations: Division of Respiratory Medicine (Amin), Department of Pediatrics, The Hospital for Sick Children; SickKids Research Institute, (Amin, Buchanan), Toronto, Ont.; The Ontario Ventilator Equipment Pool (Pizzuti), Kingston Health Sciences Centre, Kingston, Ont.; Florence Nightingale Faculty of Nursing, Midwifery and Palliative Care (Rose), King's College, London, UK

Contributors: All authors provided substantial contributions to the conception and development of this project. Reshma Amin wrote the initial draft of the manuscript, which all authors critically revised for intellectual content. The authors all approved the final version to be published and agree to be accountable for all aspects of the work.

Content licence: This is an Open Access article distributed in accordance with the terms of the Creative Commons Attribution (CC BY-NCND 4.0) licence, which permits use, distribution and reproduction in any medium, provided that the original publication is properly cited, the use is noncommercial (i.e., research or educational use), and no modifications or adaptations are made. See: https://creativecommons.org/ licenses/by-nc-nd/4.0/

Funding: This project was supported by the Ontario Ventilator Equipment Pool, operated by the Kingston Health Sciences Centre, funded by the Ontario Ministry of Health Assistive Devices Program.

Correspondence to: Reshma Amin, reshma.amin@sickkids.ca 